\title{
Longitudinal beam dynamics studies with space charge in small isochronous ring
}

\author{
E. Pozdeyev* \\ BNL, Upton, New York 11973-5000, USA \\ J. A. Rodriguez \\ CERN, Geneva, Switzerland
}

F. Marti and R. C. York

NSCL, MSU, Lansing, Michigan 48824, USA

(Received 22 January 2009; published 20 May 2009)

\begin{abstract}
Studies of the longitudinal beam dynamics in the small isochronous ring (SIR) at Michigan State University revealed a fast, space-charge driven instability that did not fit the model of the negative mass instability. The observed beam behavior can be explained by the transverse horizontal component of the coherent space-charge force and its effect on the longitudinal motion. This force effectively modifies the slip factor, shifting the isochronous point and enhancing the negative mass instability. This paper presents results of numerical and experimental studies of the longitudinal beam dynamics in SIR and proposes a simple analytical model explaining these results.
\end{abstract}

DOI: 10.1103/PhysRevSTAB.12.054202

PACS numbers: $52.59 . \mathrm{Sa}, 29.27 . \mathrm{Bd}$

\section{INTRODUCTION}

Experimental and numerical studies of space-charge effects in the small isochronous ring (SIR) conducted at Michigan State University revealed a fast, space-charge driven longitudinal instability [1,2]. The instability was observed not only in the isochronous regime but also in a machine setup with a negative slip factor (below transition). Because the beam behavior significantly differed from that predicted by the model of the negative mass instability, the cause of the instability originally was not completely understood.

The dynamics of intense beams in synchrotrons at the transition energy has been extensively studied $[3,4]$. Typically, models used for those studies included the longitudinal component of the space-charge force and its effect on the beam energy. Transverse effects were limited to the defocusing caused by the incoherent space-charge field and the corresponding effective increase of the dispersion function (Umstätter effect [5]). However, none of those models could reproduce the observed beam behavior.

The instability observed in SIR can be explained by the transverse horizontal component of the coherent spacecharge field and its effect on the coherent longitudinal motion. This field, caused by a deformation of the beam shape, enhances the negative mass instability. (We note that a similar mechanism of enhancement of the longitudinal motion by the transverse field has been observed in isochronous cyclotrons [6].) In Sec. II, we briefly describe SIR and introduce the observed instability. Using a sim-

\footnotetext{
*pozdeyev@bnl.gov
}

plified model, we demonstrate that the transverse spacecharge field can noticeably affect the longitudinal beam dynamics in the isochronous regime in Sec. III. We describe results of numerical and experimental studies of the beam dynamics in SIR in Secs. IV and V.

\section{SMALL ISOCHRONOUS RING}

The small isochronous ring (SIR) is a compact, lowenergy storage ring designed to simulate the dynamics of intense beams in the isochronous regime. The ring was developed at Michigan State University and has been operational since December 2003. Figure 1 shows a photograph of SIR. Table I gives the nominal ring parameters.

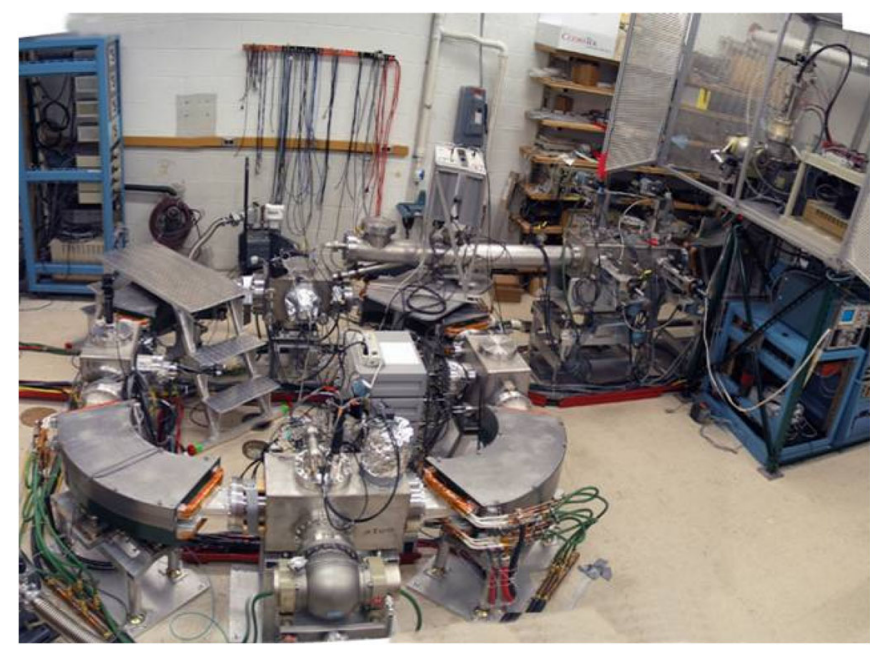

FIG. 1. (Color) Small isochronous ring. 
TABLE I. Nominal SIR parameters.

\begin{tabular}{lc}
\hline \hline Ions & $\mathrm{H}_{2}^{+}$ \\
Energy & $20 \mathrm{keV}$ \\
Beam current (peak) & $5-25 \mu \mathrm{A}$ \\
Betatron tunes $\left(\nu_{x}, \nu_{y}\right)$ & $1.14,1.11$ \\
SC tune shift $\left(\delta \nu_{x, y} @ 20 \mu \mathrm{A}\right)$ & -0.05 \\
Slip factor $\left(\alpha_{p}-1 / \gamma^{2}\right)$ & $2 \times 10^{-4}$ \\
Bunch length & $15 \mathrm{~cm}-5.5 \mathrm{~m}$ \\
Circumference & $6.6 \mathrm{~m}$ \\
Revolution period & $5 \mu \mathrm{sec}$ \\
$N_{\text {turns }}$, up to & 200 \\
\hline \hline
\end{tabular}

SIR is designed to run low-energy light-ion beams. This choice of beam parameters simplified the design of the ring and minimized the cost of the project. The ring consists of four $90^{\circ}$, zero-gradient dipole magnets with edge focusing. The edge focusing provides both isochronism and the vertical focusing. The compaction factor of the ring can be adjusted by low-field gradient correctors situated in the dipole magnets. An $\mathrm{H}_{2}^{+}$beam is produced by a multicusp ion source, that can be biased up to $30 \mathrm{kV}$. An analyzing magnet sited under the ion source provides charge-to-mass ratio selection and steers the beam towards the ring. In the injection line, the beam is truncated by a chopper and matched to the ring by a triplet of electrostatic quadrupoles. The bunch length can be changed from $15 \mathrm{~cm}$ ( $\sim 100$ nanoseconds) to $5.5 \mathrm{~m}$ ( $\sim 4$ microseconds). The beam is injected into the ring by a fast pulsed electrostatic inflector. After injection, an injected bunch coasts in the ring. Although the ring does not have rf, the bunch length stays almost unchanged because of the ring isochronism. The beam lifetime in the ring is limited to approximately 200 turns due to electron pickup from the residual gas. After a chosen number of turns, the bunch is deflected either to a phosphor screen or to a fast Faraday cup situated above and below the median plane, respectively. The Faraday cup measures the longitudinal beam profile of the beam with a time resolution of $1 \mathrm{~ns}$ corresponding to a spatial resolution of approximately $1.5 \mathrm{~mm}$. The phosphor screen is used to observe the transverse beam profile. Changing the extraction time, we can observe temporal evolution of the longitudinal and radial beam profiles in the ring.

Figure 2 shows the longitudinal bunch profile measured by the Faraday cup right after injection and after turns 10 and 20 . The beam exhibits a longitudinal instability, causing breakup of injected bunches in 20 turns. The longitudinal breakup is accompanied by a growth of the normalized energy spread from $\sim 10^{-4}$ to $\sim 0.05$. Numerical simulations, including only the space-charge field, show a remarkable agreement with the experiment (for details, see Sec. V). Experimental results presented later in this paper show that the growth rate of the instability in the isochronous regime scales linearly with the beam current.

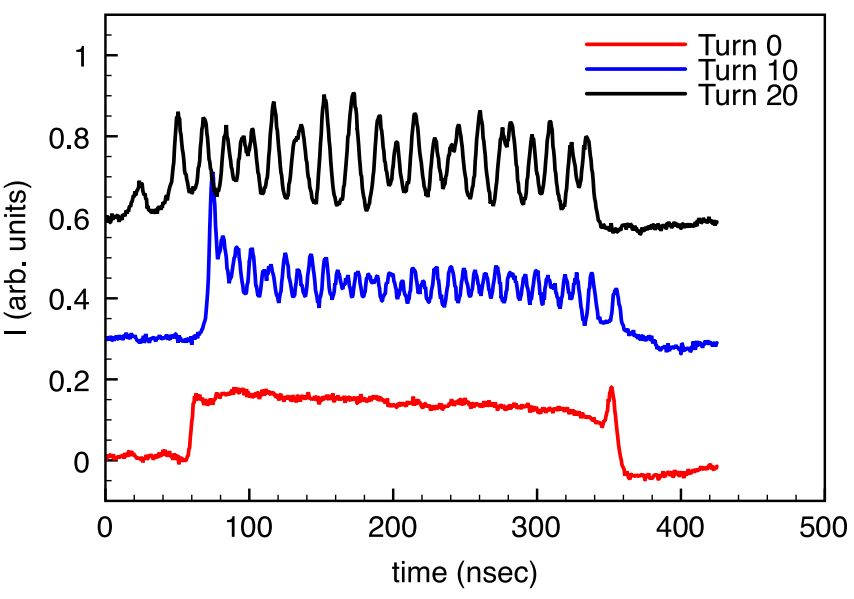

FIG. 2. (Color) Longitudinal bunch profiles measured by the fast Faraday cup after injection and after turns 10 and 20. The profiles measured after 10 and 20 turns are shifted vertically by 0.3 and 0.6 , respectively. In these measurements, the beam energy was $20.9 \mathrm{MeV}$ and the peak current was $9.3 \mu \mathrm{A}$.

\section{ENHANCEMENT OF NEGATIVE MASS INSTABILITY}

In this section, we demonstrate that the longitudinal space-charge impedance has a maximum at a wavelength comparable to the beam diameter. An energy perturbation with a short wavelength causes a horizontal offset of the beam centroid that induces a transverse electric field. This field effectively modifies the slip factor, shifting the isochronous point and enhancing the negative mass instability.

\section{A. Longitudinal space-charge impedance: short wavelength approximation}

The space-charge impedance (per turn) in the long wavelength limit is given by

$$
Z_{\|}(k)=i k \frac{Z_{0} R_{0}}{\gamma^{2} \beta}\left[\frac{1}{2}-\ln \left(\frac{a}{b}\right)\right],
$$

where $Z_{0}$ is the characteristic vacuum impedance, $377 \Omega$, $R_{0}$ is the average machine radius, $\gamma$ and $\beta$ are the relativistic factors, and $a$ and $b$ are the beam and the vacuum chamber radii, respectively. Equation (1) assumes a beam with a uniform cross section.

Equation (1) becomes invalid if the wavelength becomes comparable to the diameter of the vacuum chamber. In the short wavelength limit, the effect of image charges can be neglected and the field on the beam axis can be obtained by simple integration. For a round beam with a uniform transverse distribution, this yields the longitudinal spacecharge impedance in the short wavelength limit:

$$
Z_{\|}(k)=i \frac{2 Z_{0} R_{0}}{k a^{2} \beta}\left[1-\frac{k a}{\gamma} \cdot K_{1}\left(\frac{k a}{\gamma}\right)\right],
$$




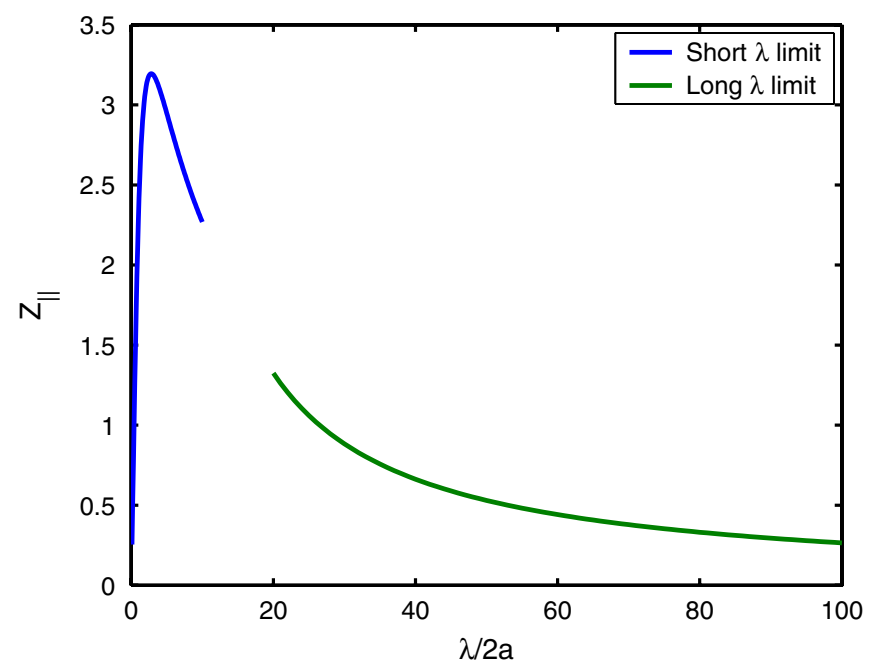

FIG. 3. (Color) Longitudinal impedance (arbitrary units) in long and short wavelength approximations as a function of the wavelength normalized to the beam diameter, $2 a$. The impedance reaches its maximum at $\lambda \approx 5 a$. The $b / a$ ratio is equal to 5 .

where $K_{1}$ is the modified Bessel function of the second kind.

Figure 3 shows the longitudinal space-charge impedance in both long and short wavelength limits for a nonrelativistic beam $(\gamma=1)$ and the ratio $b / a$ equal to 5 . The impedance reaches its maximum value at the wavelength approximately equal to 2.5 beam diameters.

\section{B. Coherent transverse space-charge field}

We will use the quantities $x$ and $y$ to denote the horizontal and vertical displacements from a reference orbit and $z$ to denote the longitudinal coordinate relatively to a reference particle moving on the reference trajectory.

If the longitudinal linear particle density of a coasting beam is given by $n(z)=n_{0}+n_{1} \sin (k z)$, the beam induces a longitudinal field proportional to $n_{1} \cos (k z)$. This field introduces a coherent energy modulation. As the energy of particles changes, their transverse position also changes because of the dispersion. Accompanying these horizontal wiggles of the beam centroid is a transverse horizontal electric field (see Fig. 4). This field is generated by two sources: (a) interaction between radially shifted parts of the beam and (b) image charges induced on the vertical (side) walls of the vacuum chamber. In calculating the coherent field, we assume that: (i) The local curvature of the beam orbit has a small effect on the space-charge field and can be neglected. (ii) The perturbation of the linear charge density is small comparatively to the average charge density $\left(n_{0} \gg\right.$ $n_{1}$ ). (iii) The distortion of the horizontal beam shape is small. That is, we consider only the initial stage of the instability. (iv) The coherent transverse force is caused only by the interaction between shifted parts of the beam. We neglect the field of image charges because of a large aspect ratio of the SIR vacuum chamber $(11 \mathrm{~cm}$ horizontal

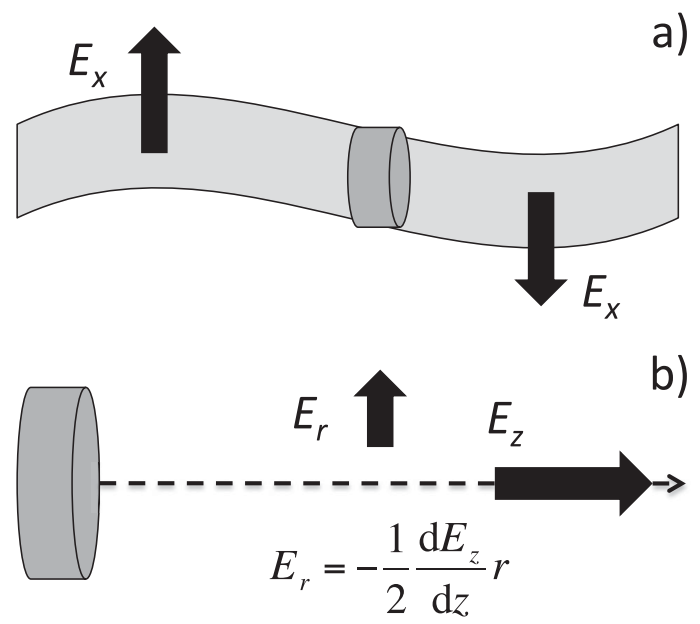

FIG. 4. (a) Schematic drawing of a horizontal beam shape distortion caused by energy modulation. Also, the sketch depicts the $x$ component of the coherent field and one of the beam slices that are used to calculate this field. (b) A schematic drawing of a cylindrical beam slice with its longitudinal and transverse fields.

to $4.5 \mathrm{~cm}$ vertical). (v) The beam cross section is axially symmetric (round), which is a good first approximation for SIR [1]. (vi) The beam is nonrelativistic $(\gamma=1)$, which is a valid assumption for SIR.

The $x$ component of the coherent field can be calculated as an integral over contributions produced by thin cylindrical slices of the beam (see Fig. 4). Calculating the field is greatly simplified if the amplitude of the wiggles is small and only the linear term of the field is calculated. In the first approximation, the radial field produced by an infinitesimally thin, uniformly charged slice at a distance $r$ from the cylinder axis is given by

$$
E_{r}^{(\text {slice })}(r, z)=-\frac{1}{2} \frac{d E_{z}^{(\text {slice })}}{d z} r=-\frac{d q}{\left(a^{2}+z^{2}\right)^{3 / 2}} r,
$$

where $a$ is the radius of the beam cross section and $d q$ is the charge of the slice, $d q=\rho \pi a^{2} d z$. Assuming that the horizontal offset of the beam centroid is given by $x_{c}(z)=$ $a_{c} \cos (k z)$, where $k$ is the wave number of the perturbation, we can write the $x$ component of the field at an arbitrary point with coordinates $(x, z)$ as

$$
\begin{aligned}
E_{x}(x, z) & =\pi \rho a^{2} \int_{-\infty}^{\infty} \frac{\left[x-a_{c} \cos (k \bar{z})\right] d \bar{z}}{\left[a^{2}+(z-\bar{z})^{2}\right]^{3 / 2}} \\
& =2 \pi \rho\left[x-x_{c}(z) k a \cdot K_{1}(k a)\right] .
\end{aligned}
$$

\section{Dispersion function and slip factor with space charge}

To simplify our treatment of the beam dynamics with the space-charge field, we adopt the smooth approximation. We also assume no incoherent energy spread. The equation of the radial motion in the smooth approximation and the low-energy limit can be written as 


$$
x^{\prime \prime}+\frac{\nu^{2}}{R_{0}^{2}} x=\frac{1}{R_{0}} \frac{\delta p}{p}+\frac{e E_{x}}{m \beta^{2} c^{2}},
$$

where $e$ is the elementary charge, $c$ is the speed of light, $\nu$ is the bare betatron tune, and $E_{x}$ is given by (4). As will be shown later, the instability in SIR develops on a time scale of ten turns, which is much slower than the period of betatron oscillations. Therefore, the particle motion can be represented as a sum of a betatron term and a term representing the slow motion of the beam centroid: $x=$ $x_{c}+x_{\beta}$. Substituting this and (4) into (5) yields equations for the betatron oscillations and the beam centroid:

$$
\begin{gathered}
x_{\beta}^{\prime \prime}+\frac{\nu^{2}}{R_{0}^{2}} x_{\beta}=\frac{2 e \pi \rho}{m \beta^{2} c^{2}} x_{\beta}, \\
x_{c}^{\prime \prime}+\frac{\nu^{2}}{R_{0}^{2}} x_{c}=\frac{1}{R_{0}} \frac{\delta p}{p}+\frac{2 e \pi \rho\left[1-k a \cdot K_{1}(k a)\right]}{m \beta^{2} c^{2}} x_{c} .
\end{gathered}
$$

Equation (6) is the equation of the betatron motion with the incoherent space force. Equation (7) is the equation of motion of the beam centroid. The expression $2 e \pi \rho x_{c}[1-$ $\left.k a \cdot K_{1}(k a)\right]$ in the second term on the right-hand side of (7) is the electric field on the beam centroid, induced by the coherent distortion of the beam shape. Figure 5 shows the factor $1-k a \cdot K_{1}(k a)$ as a function of the wavelength. We note that the amplitude of $E_{x}$ on the beam centroid reaches its maximum at wavelengths smaller than the beam diameter and vanishes in the long wavelength limit.

An off-momentum closed orbit solution of Eq. (7) easily can be found:

$$
x_{\mathrm{ss}} \approx \frac{R_{0}}{\nu^{2}}\left[1+2\left(\frac{-\delta \nu}{\nu}\right)_{\mathrm{sc}}\left[1-k a \cdot K_{1}(k a)\right]\right] \frac{\delta p}{p},
$$

where we formally expressed the uniform charge density $\rho$ via the incoherent space-charge tune shift to simplify for-

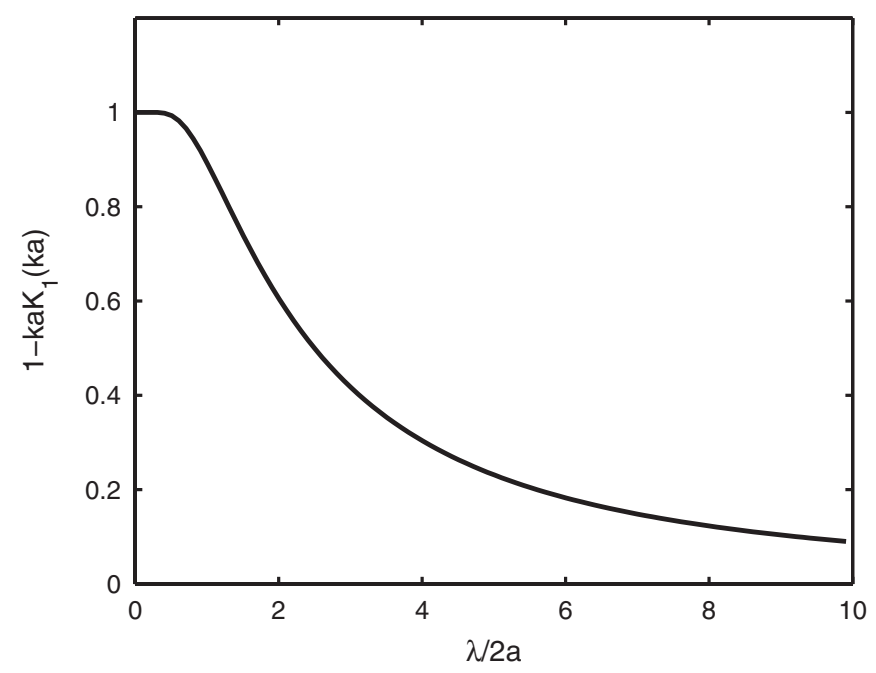

FIG. 5. Factor $1-k a \cdot K_{1}(k a)$ as a function of the wavelength normalized to the beam diameter, $2 a$. mulas:

$$
\frac{\pi e \rho}{m \beta^{2} c^{2}}=\frac{\nu^{2}}{R_{0}^{2}}\left(\frac{-\delta \nu}{\nu}\right)_{\mathrm{sc}}
$$

Thus, the coherent space-charge field changes the dispersion function $D$ by

$$
\delta D \approx 2 D\left(\frac{-\delta \nu}{\nu}\right)_{\mathrm{sc}}\left[1-k a \cdot K_{1}(k a)\right] .
$$

This dispersion function variation changes the slip factor:

$$
\begin{aligned}
\eta & =\frac{p}{T} \frac{d T}{d p}=\eta_{0}+\frac{\delta D}{R_{0}} \\
& \approx \eta_{0}+2\left(\frac{-\delta \nu}{\nu}\right)_{\mathrm{sc}}\left[1-k a \cdot K_{1}(k a)\right],
\end{aligned}
$$

where $\eta_{0}$ is the bare slip factor without the space charge. Because the incoherent tune shift is negative, the space charge tends to increase the dispersion function and the slip factor. In the isochronous regime, $\eta_{0}$ is zero by definition. Thus, if the machine optics is exactly isochronous, the effective slip factor with the space charge is

$$
\eta \approx 2\left(\frac{-\delta \nu}{\nu}\right)_{\mathrm{sc}}\left[1-k a \cdot K_{1}(k a)\right] .
$$

\section{Instability growth rate.}

The growth rate of the microwave instability is given by the formula [4]

$$
\tau^{-1}(k)=\omega_{0} \sqrt{-i \frac{\eta e I_{0} k R_{0} Z_{\|}}{2 \pi \beta^{2} E}},
$$

where $\omega_{0}$ is the revolution angular frequency, $I_{0}$ is the average peak current, and $E$ is the beam full energy.

If $Z_{\|}$is the space-charge impedance, the microwave instability is also referred to as the negative mass instability. The negative mass instability can occur only if $\eta$ is positive. As it was shown before, the coherent component of the space-charge force effectively increases the dispersion and the slip factor of the coherent motion. Thus, a negative-mass-like instability can happen even if the bare machine optics is set below the transition. The growth rate of this instability can be found by substituting (11) into (13). Because the longitudinal space-charge impedance peaks at a short wavelength, we can use the short wavelength approximation given by (2).

If the machine bare optics is exactly isochronous $\left(\eta_{0}=\right.$ $0)$, the growth rate is given by

$$
\tau^{-1}(k) \approx 2 \sqrt{2} \omega_{0}\left(\frac{-\delta \nu}{\nu}\right)_{\mathrm{sc}}\left[1-k a \cdot K_{1}(k a)\right] .
$$

Thus, the growth rate of this instability at the transition is proportional to the beam peak current. 
If the bare machine optics is set below the transition $\left(\eta_{0}<0\right)$, the instability has a threshold current corresponding to the condition

$$
2\left(\frac{\delta \nu}{\nu}\right)_{\mathrm{sc}} \approx \eta_{0} .
$$

Below transition, the maximum growth rate depends on the beam current $I$ as

$$
\tau_{\max }^{-1} \sim I \sqrt{1-\frac{I_{\mathrm{th}}}{I}},
$$

where $I_{\mathrm{th}}$ is the threshold corresponding to the condition (15).

\section{E. Suppression of growth of short wavelength harmonics}

Particles executing betatron oscillations also oscillate longitudinally within the beam. This longitudinal motion is due to coupling between the horizontal and longitudinal degrees of freedom, described by the Hamiltonian term $\epsilon x / R$, where $\epsilon$ is the energy deviation and $R$ is the curvature of the reference beam trajectory. Because the growth time of the instability in SIR is longer than the period of betatron oscillations, the energy of particles is affected by the longitudinal force averaged over these fast longitudinal oscillations. Similarly, the transverse coherent field is averaged over the longitudinal oscillations. In SIR, the amplitude of these longitudinal oscillations is approximately equal to the amplitude of the betatron oscillations. Therefore, the field of harmonics with a wavelength shorter than the transverse size of the beam is averaged out. This averaging suppresses the growth of short wavelength harmonics. Using these arguments and Eq. (14), we can expect that harmonics with a wavelength approximately equal to or somewhat larger than the beam diameter have to exhibit the strongest growth.

\section{SIMULATIONS OF BEAM DYNAMICS IN SIR}

We used the code CYCO [1] to simulate the beam dynamics in SIR. The code employs the classical 4th order Runge-Kutta method to calculate trajectories of macroparticles in a 3D magnetic field map, solving the full, unsimplified system of equations of motion with the space charge. The 3D space-charge field of the beam is calculated by a particle-in-cell field solver. In calculating the space-charge field, the program includes the field of image charges, assuming that the vacuum chamber consists of two perfectly conducting planes parallel to the median plane. Every time the code calculates the space-charge field, it modifies the momentum of each particle by a product of the space-charge force and the time interval between field calculations (impulse approximation). No other collective field besides the space charge is included in the code. In simulating the beam dynamics in SIR, the space-charge field was calculated 90 times per revolution. The number of macroparticles was $3 \times 10^{5}$.

The initial beam distribution mimicked the phase space of the actual beam measured by two pairs of moving slits and the fast Faraday cup. The transverse distribution was a 4D uniform distribution in $x, x^{\prime}, y, y^{\prime}$ :

$$
f=H\left(1-\frac{A_{x}}{\epsilon_{x}}-\frac{A_{y}}{\epsilon_{y}}\right),
$$

where $H$ is the Heaviside step function and $A_{x}$ and $A_{y}$ are Courant-Snyder invariants. The maximum values of $A_{x}$ and $A_{y}$ were the same, $\epsilon_{x, y}=5 \times 10^{-5} \mathrm{~m}$, corresponding to the beam diameter in the ring approximately equal to $1 \mathrm{~cm}$. The initial longitudinal distribution was uniform. For most of our simulations, we used a $40 \mathrm{~cm}$ long bunch with hard edges. The initial energy spread was zero. Multicusp ion sources typically produce an energy spread of a few $\mathrm{eV}$, yielding a normalized initial energy spread of $\sim 10^{-4}$ in the

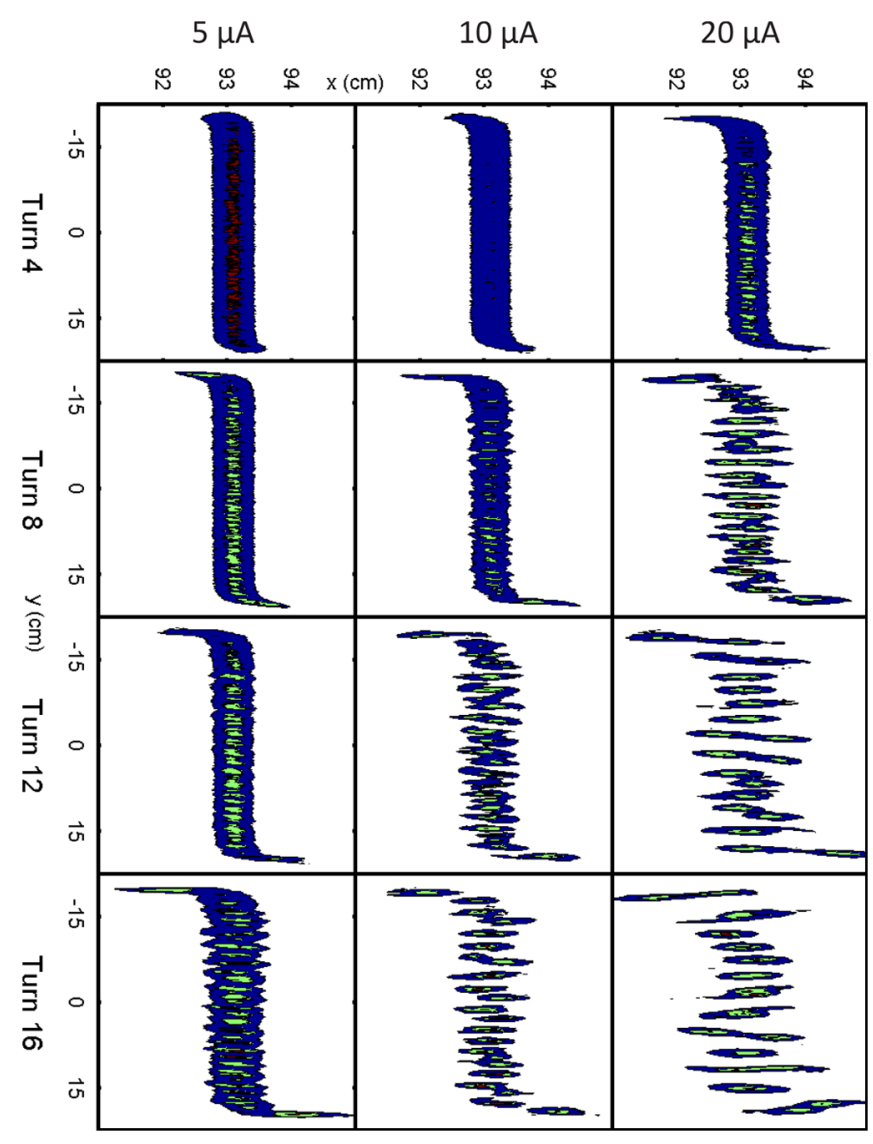

FIG. 6. (Color) Results of simulations of the beam dynamics in SIR for three different peak intensities: 5, 10, and $20 \mu \mathrm{A}$. The bare machine optics is isochronous. Each frame contains a projection of the bunch charge density on the median plane for a given turn and intensity. The vector of velocity is directed from the top of the figure to the bottom. The horizontal axis of each frame corresponds to $x$ measured in centimeters from the center of the ring. The vertical axis is along the beam trajectory. The size of each frame is $5(x) \times 45(z) \mathrm{cm}^{2}$. 
case of SIR. Because of this, the initial energy spread was completely neglected in the simulations.

The beam dynamics in SIR was simulated in two regimes: (i) Isochronous. - In this case, the slip factor in the SIR computer model was adjusted to match the measured slip factor of SIR, $+2 \times 10^{-4}$. (ii) Below transition (negative slip factor).-To simulate the beam dynamics below transition the distance between the SIR dipoles was increased in the computer model by $9 \mathrm{~cm}$ that yielded a slip factor of -0.04 . Although the slip factor in SIR is adjusted by gradient correctors in the dipoles magnets, changing the drift length in the computer model is easier than recalculating the magnetic field.

\section{A. Space-charge driven beam instability}

Simulations of the beam dynamics in the isochronous regime demonstrated that the space-charge force excites a fast beam instability that ultimately causes fragmentation of bunches into small clusters. This instability is accompanied by a fast growth of the energy spread. Figure 6 shows the instability for three different beam intensities.

Figure 7 shows evolution of the linear charge density in the central part of a bunch with a peak current of $10 \mu \mathrm{A}$. The charge density is shown for turns 7,11 , and 15 . Figure 8 shows the fast-Fourier-transform spectrum of the profiles shown in Fig. 7. We note that harmonics with $\lambda \approx 1.5 \mathrm{~cm}$ exhibit the strongest growth while harmonics with the wavelength shorter than $0.5 \mathrm{~cm}$ show no growth. As described in Sec. IIIE, this suppression is caused by averaging out the field of short wavelength harmonics due to fast longitudinal oscillations of particles within the beam.

Figure 9 shows the logarithm of the amplitude of five different harmonics with wavelengths $0.6,0.75,1,1.5$, and

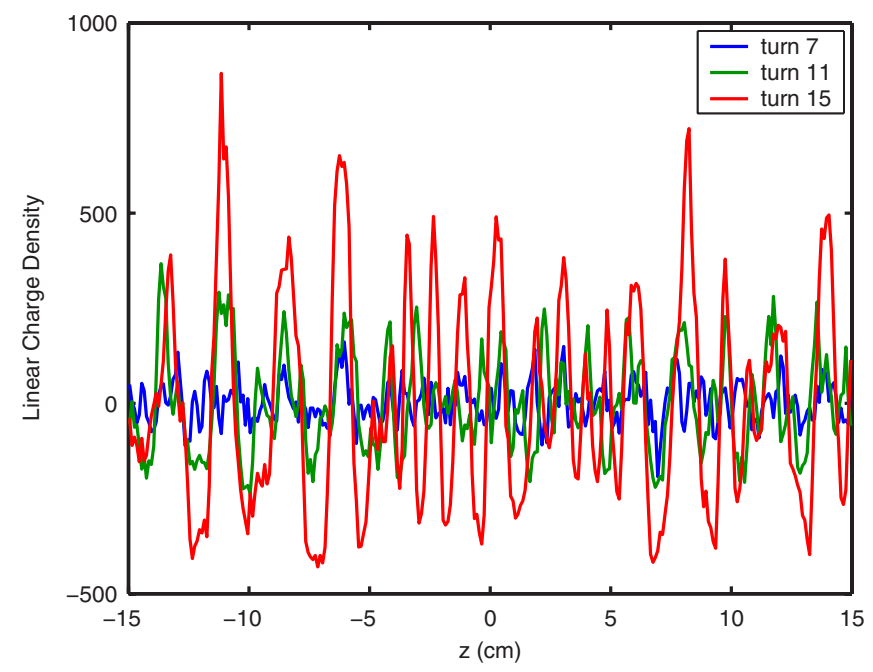

FIG. 7. (Color) Linear particle density profiles for turns 7, 11, and 15 . The average level equal to 430 was subtracted. Only the central $\pm 15 \mathrm{~cm}$ part of a $40 \mathrm{~cm}$ long bunch is shown. The charge density is in arbitrary units.

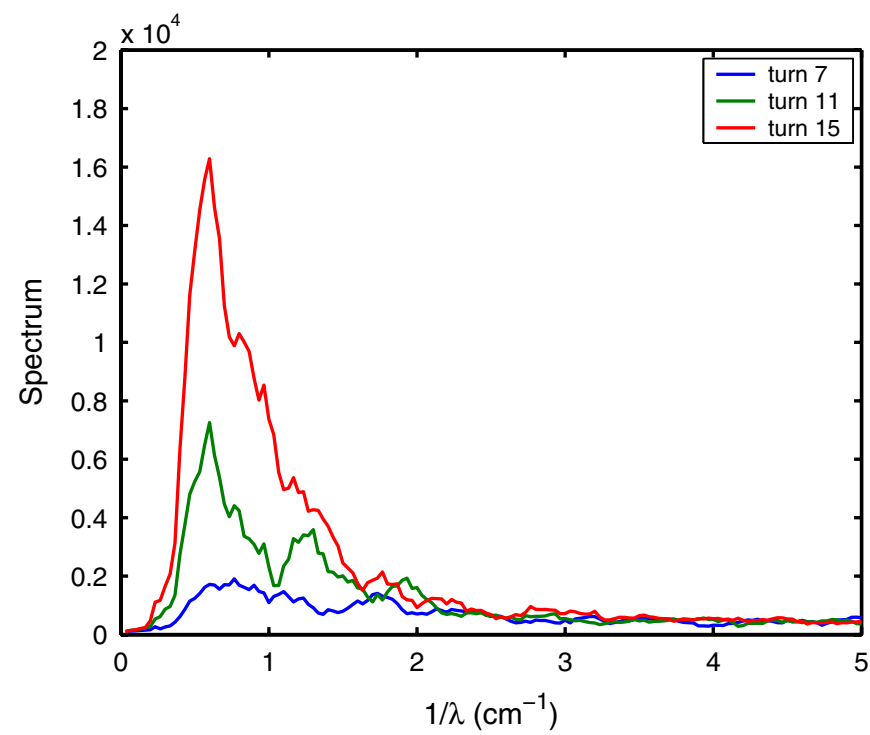

FIG. 8. (Color) Spectra of linear charge density profiles shown in Fig. 7.

$3 \mathrm{~cm}$ as a function of turn number. All the curves with the exception of the harmonic with $\lambda=3 \mathrm{~cm}$ show an exponential growth starting from turn 1 up to turn 11 or 12 . The harmonic with $\lambda=1.5 \mathrm{~cm}$ exhibits the highest growth rate. After turn 12, the growth saturates. This saturation corresponds to almost complete breakup of the bunch into small separated clusters (see Fig. 6, column 2, turns 12 and 16, also see Fig. 7). The amplitude of the harmonic with $\lambda=3 \mathrm{~cm}$ starts growing after turn 5 and does not exhibit saturation up to turn 15 .

\section{B. Scaling with the beam current}

First, we simulated the beam dynamics in the isochronous SIR optics. Figure 10 shows the growth rate of the

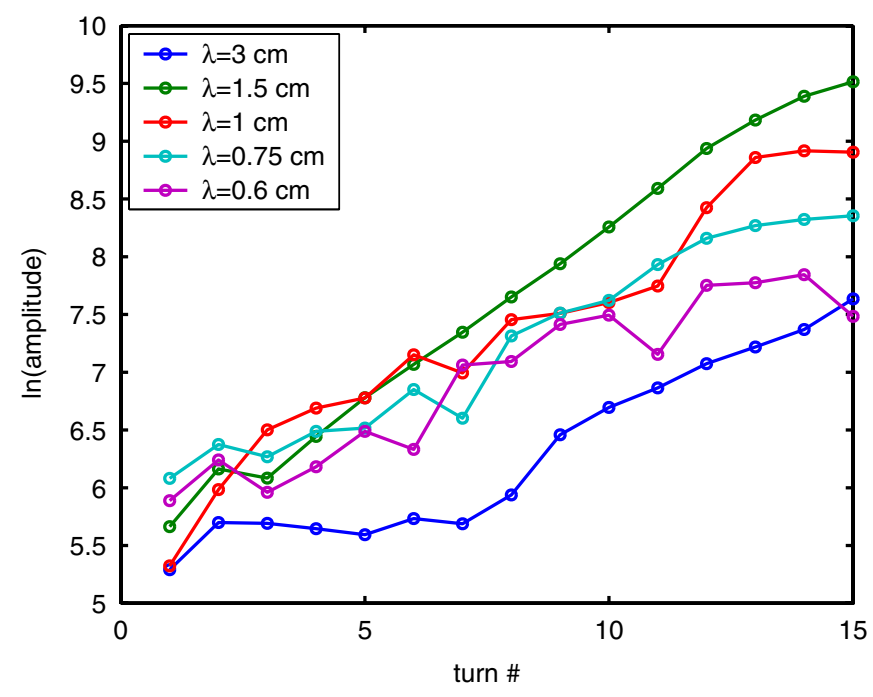

FIG. 9. (Color) Logarithm of the amplitude of harmonics with $\lambda=0.6,0.75,1,1.5$, and $3 \mathrm{~cm}$ as a function of turn number. The harmonic with $\lambda=1.5 \mathrm{~cm}$ exhibits the highest growth rate. 


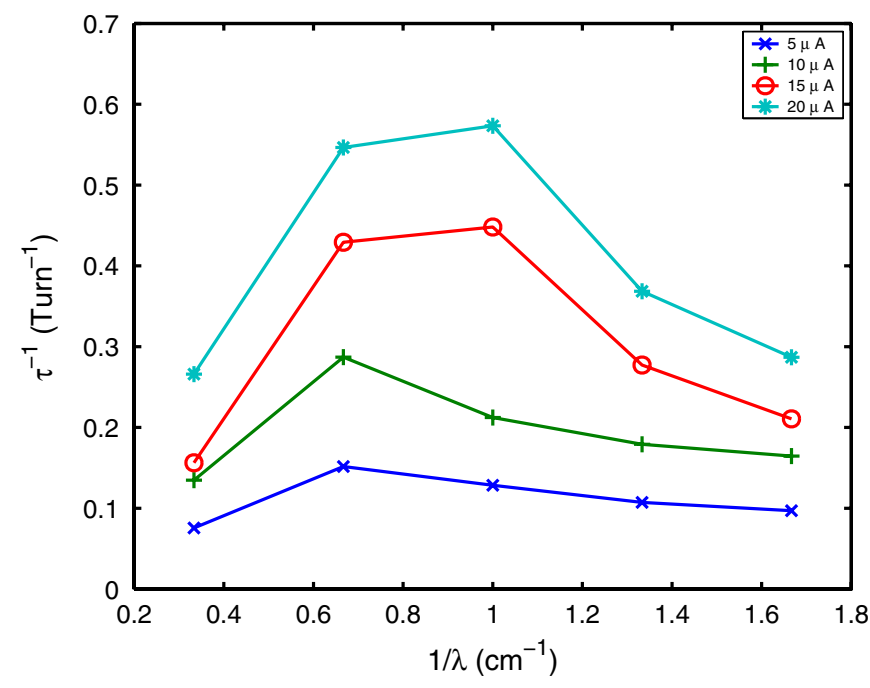

FIG. 10. (Color) Amplitude growth rate of harmonics $\lambda=0.6$, $0.75,1,1.5$, and $3 \mathrm{~cm}$. The growth rate shown for four different peak intensities: 5, 10, 15, and $20 \mu \mathrm{A}$.

mentioned above harmonics for four different beam intensities: 5, 10, 15, and $20 \mu \mathrm{A}$. Figure 11 shows the same growth rates but normalized to the beam current. The normalized curves practically overlap in the region $\lambda=$ $1.5 \mathrm{~cm}$ and are close to each other in other regions. Thus, Fig. 11 demonstrates that the growth rate of the instability in the isochronous regime scales linearly with the beam current. This contradicts the classical model of the negative mass instability and can be explained only if the $x$ component of the coherent space-charge field is included. Also, the simulated growth rates significantly exceed those predicted by the negative mass instability model. Table II shows the simulated growth rate of the harmonic $\lambda=1.5$

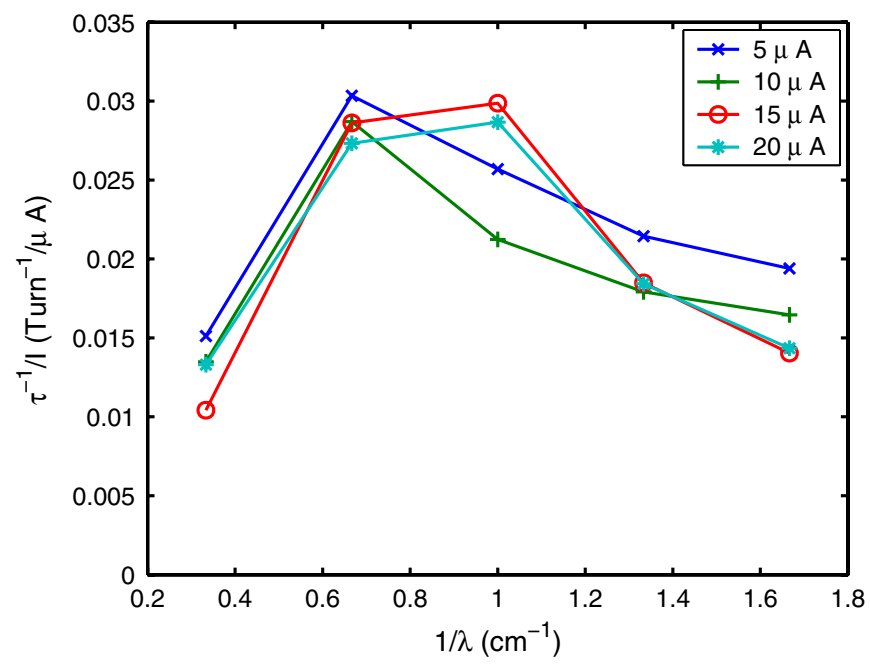

FIG. 11. (Color) Amplitude growth rate for harmonics with the wavelength $\lambda=0.6,0.75,1,1.5$, and $3 \mathrm{~cm}$ normalized to the peak beam current. The normalized growth rate shown for four different peak intensities: 5, 10, 15, and $20 \mu \mathrm{A}$.
TABLE II. Amplitude growth rate of the harmonic $\lambda=$ $1.5 \mathrm{~cm}$ observed in simulations (second column), predicted by Eq. (14) for $a=0.5 \mathrm{~cm}$ and $\lambda=1.5 \mathrm{~cm}$ (third column), and given by the conventional microwave instability formula, Eq. (13), with the constant slip factor $\eta_{0}=+2 \times 10^{-4}$ (fourth column).

\begin{tabular}{lccc}
\hline \hline & \multicolumn{3}{c}{$\tau^{-1}\left(\operatorname{turn}^{-1}\right)$} \\
$I_{0}(\mu \mathrm{A})$ & Simulated & Eq. (14) & $\begin{array}{c}\text { Eq. (13) } \\
\eta_{0}=2 \times 10^{-4}\end{array}$ \\
\hline 5 & 0.15 & 0.18 & 0.02 \\
10 & 0.29 & 0.35 & 0.028 \\
15 & 0.43 & 0.52 & 0.034 \\
20 & 0.55 & 0.7 & 0.038 \\
\hline \hline
\end{tabular}

and that calculated using Eqs. (2) and (13) and the constant slip factor $\eta_{0}=2 \times 10^{-4}$. Also, the table shows the growth rate given by Eq. (14) for $a=0.5 \mathrm{~cm}$ and $\lambda=$ $1.5 \mathrm{~cm}$. According to the table, Eq. (14) predict growth rates approximately $20 \%$ higher than those observed in the simulations.

Below transition $\left(\eta_{0}=-0.04\right)$, bunches with a peak current of $5 \mu \mathrm{A}$ exhibited no instability during 100 turns, the maximum number of simulated turns. The other three simulated cases with a peak current of 10,15 , and $20 \mu \mathrm{A}$ exhibited the instability and clustering. Figure 12 shows the amplitude growth rate of the harmonic with $\lambda=$ $1.5 \mathrm{~cm}$ as a function of the beam current. The curve $y=$ $0.028 x \sqrt{1-7 / x}$ fits well the simulation results, predicting

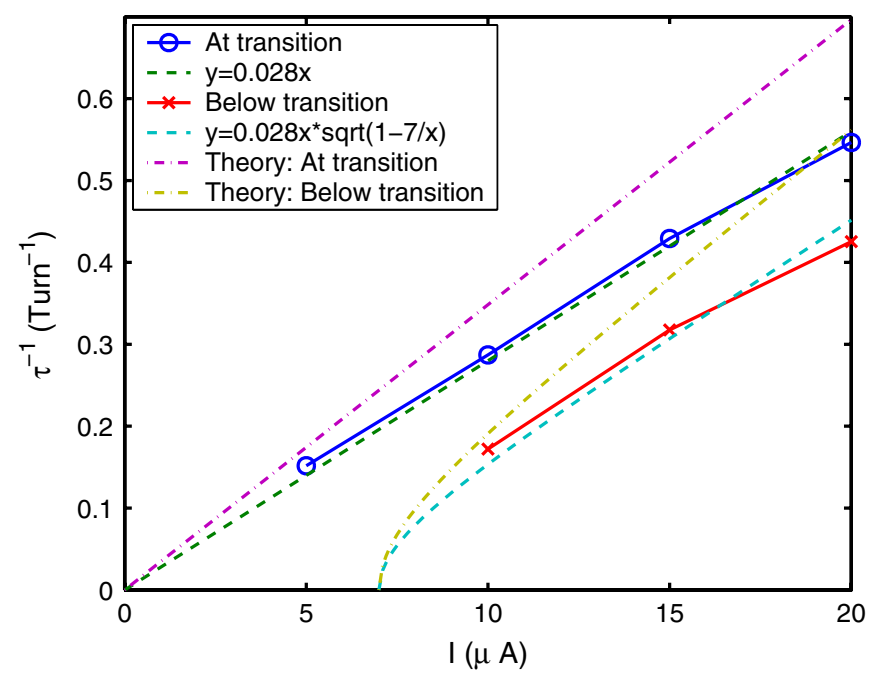

FIG. 12. (Color) Growth rate of the harmonic with $\lambda=1.5 \mathrm{~cm}$ for the isochronous optics and the optics with the negative slip factor -0.04 . The simulated data (solid lines with points) is fitted with corresponding fits: $y=0.028 x$ and $y=$ $0.028 x \sqrt{1-7 / x}$. Additionally, growth rates calculated directly from Eqs. (14) and (16) for $a=0.5 \mathrm{~cm}$ and $\lambda=1.5$ are shown. The growth rates predicted by the model exceed the simulated growth rates by approximately $20 \%$. 
a threshold of $7 \mathrm{~mA}$. Also, Fig. 12 shows the amplitude growth rate for the isochronous case and the linear fit $y=$ $0.028 x$. The good agreement between the simulation results and the fits indicates that the proposed model of the instability correctly predicts scaling with the beam current. Additionally, Fig. 12 shows the growth rates calculated directly from (14) and (16) for $a=0.5 \mathrm{~cm}$ and $\lambda=$ $1.5 \mathrm{~cm}$. The incoherent space-charge tune shift used to calculate the theoretical growth rates was estimated using a threshold current of $7 \mathrm{~mA}$ and Eq. (15).

\section{EXPERIMENTAL STUDIES OF BEAM DYNAMICS IN SIR}

In this section, we present results of experimental studies of space-charge effects in SIR. The experimental procedure is described in Sec. II. These studies were conducted in a near-isochronous regime. The SIR slip factor $\eta_{0}$ was measured by the time of flight technique that yielded $\eta_{0}=+2 \times 10^{-4}$.

To quantify the instability we counted the number of peaks of the longitudinal charge density measured by the Faraday cup [2]. The density peaks were defined relatively to a chosen level of the initial charge density. Using this observable allowed us to compare simulation results to
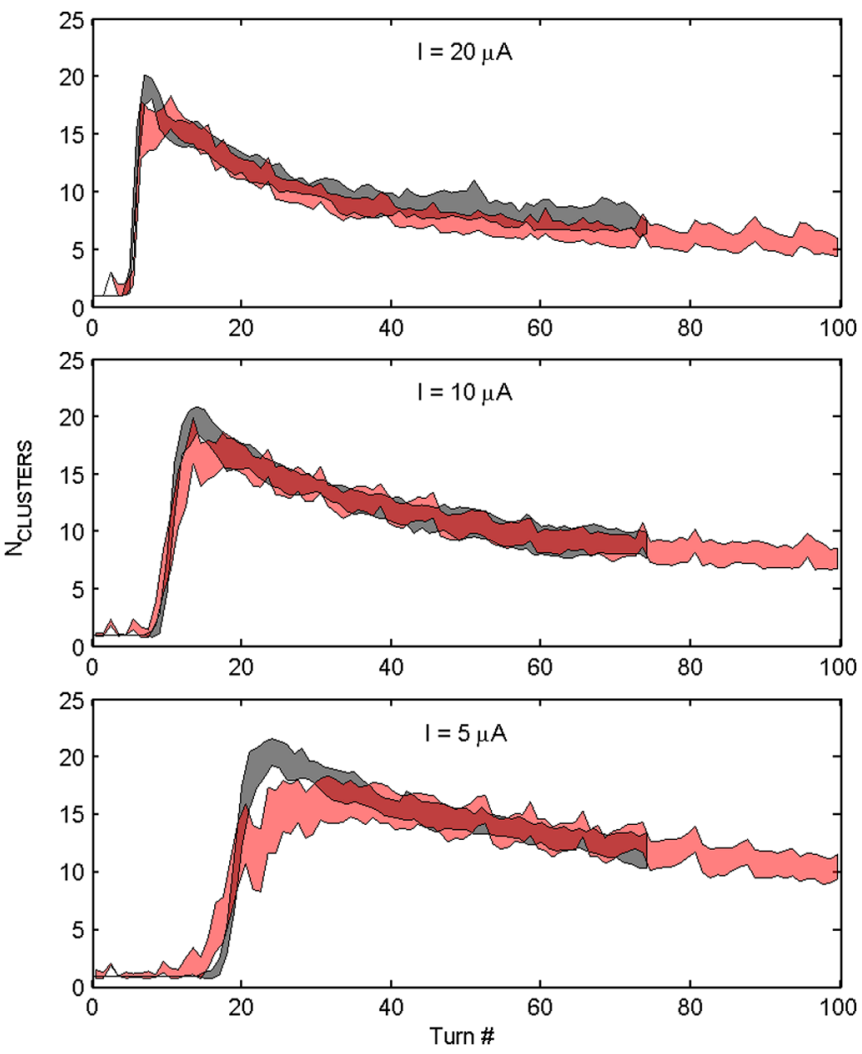

FIG. 13. (Color) Simulated (in grey) and experimentally measured (in red) number of linear charge density peaks vs turn number for three different beam intensities: 5, 10, and $20 \mu \mathrm{A}$. Experimental and simulated data closely overlap. experimental data and study scaling of the instability with beam parameters. Observing evolution of the beam spectrum could give us more details about the beam dynamics in SIR. However, in this paper, we present the original experimental results obtained by the peakcounting technique.

\section{A. Comparison of simulation results to experimental data}

Figure 13 shows the simulated and experimentally measured number of linear charge density peaks as a function of turn number for three different beam intensities. The level at which these peaks were counted was set to $50 \%$ of the initial density. The simulation and experimental results are overlaid on top of each other. The simulation data shows an average number of peaks corresponding to 14 different randomly seeded distributions. The width of the curves corresponds to 2 standard deviations of the number of peaks. The experimental data shows the result of processing of 100 distributions for each turn. According to the figure, the simulation results agree remarkably well with the experimental data.

\section{B. Scaling with the beam current}

Figure 14(a) shows the measured number of peaks as a function of turn number for three different beam intensities. Figure 14(b) depicts the same data plotted vs a product of turn number and beam intensity. The overlap of the curves corresponding to different beam intensities in Fig. 14(b) confirms the linear dependence of the instability growth rate on the beam current.
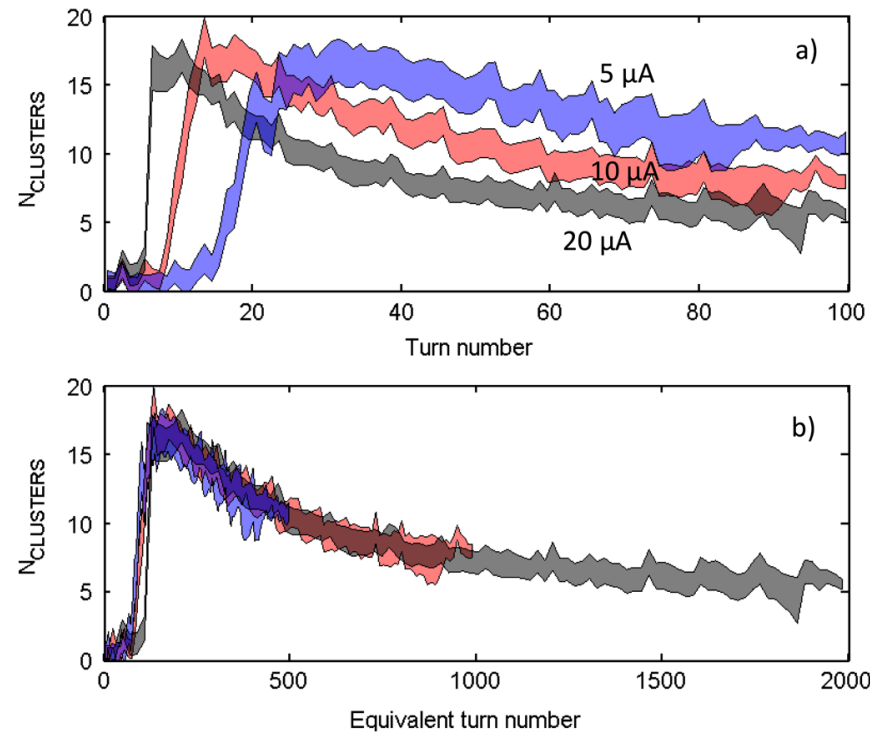

FIG. 14. (Color) (a) The number of peaks of the linear charge density as a function of turn number for three different peak intensities: 5, 10, and $20 \mu \mathrm{A}$. (b) The same data plotted as a function of a product of turn number and beam intensity (equivalent turn number). 

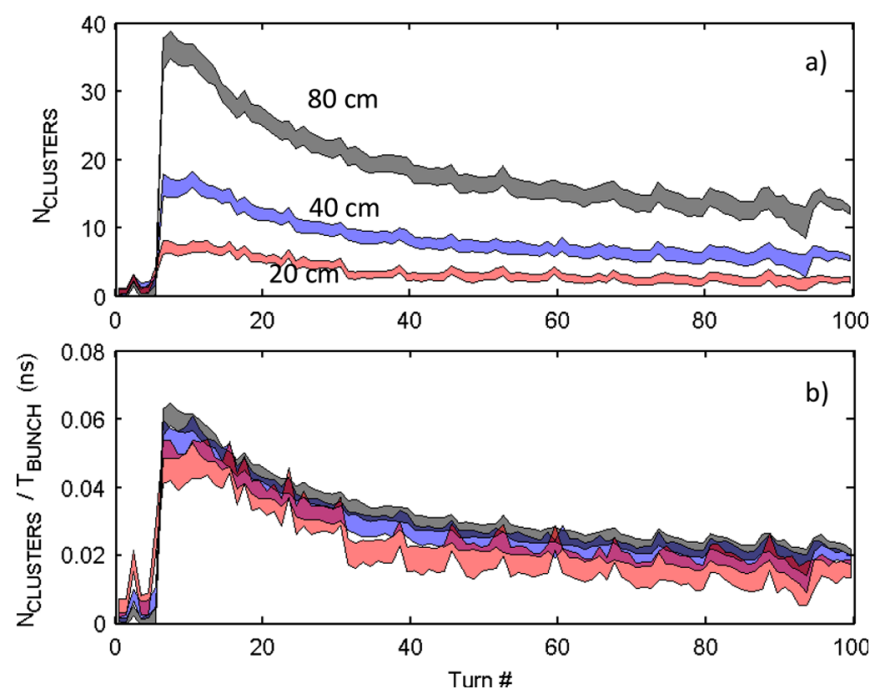

FIG. 15. (Color) (a) The number of peaks of the linear charge density for three different initial bunch lengths: 20, 40, and $80 \mathrm{~cm}$. (b) The same data normalized to the initial bunch length measured in nanoseconds. It is obvious that the growth rate of the instability does not depend on the bunch length.

\section{Scaling with the bunch length}

Figure 15(a) shows evolution of the measured number of peaks for three different bunch lengths. If the number of peaks is divided by the initial bunch length, all three curves practically overlap as it is depicted in Fig. 15(b). This indicates that the instability happens simultaneously throughout a bunch, not only at the bunch edges where the space-charge field is strongest. This result also shows that the size of clusters does not depend on the bunch length.

\section{SUMMARY}

The model presented in the paper proposes an enhancement mechanism of the negative mass instability in the isochronous regime. According to the model, (i) the transverse electric field caused by a coherent deformation of the beam shape effectively increases the dispersion function and the slip factor, enhancing the negative mass instability in the isochronous regime. (ii) The instability growth rate in the isochronous regime scales linearly with the beam peak current. (iii) The instability has a threshold given by (15) if the slip factor is negative. In this case, the dependence of the growth rate on the beam peak current is given by (16). (iv) Harmonics with a wavelength approximately equal to or somewhat larger than the beam diameter exhibit the strongest growth.

The model describes the simulated and experimentally observed beam behavior in SIR with a reasonable accuracy, especially considering the simplistic nature of the analysis presented in the paper. To be applicable to large scale, high energy machines, the described model has to be extended to include relativistic effects. Additionally, the Landau damping caused by the incoherent energy spread has to be included in the model.

\section{ACKNOWLEDGMENTS}

We would like to thank D. Deveraux, R. Fontus, D. Sanderson, A. Zeller, and R. Zink for extensive help during the design and construction of SIR. We would like to thank the personnel of the NSCL shop for their work on SIR components. Also, we would like to thank Lawrence Berkeley National Laboratory for lending us the multicusp source used in SIR.

[1] E. Pozdeyev, Ph.D. thesis, Michigan State University, 2003.

[2] J. A. Rodriguez, Ph.D. thesis, Michigan State University, 2004.

[3] S. A. Bogacz, in Proceedings of the 2nd EPAC, Nice, France (Editions Frontieres, Gif-sur-Yvette, France, 1990), p. 1524.

[4] K. Y. Ng, Technical Report No. Fermilab-FN-0713, FNAL, 2002.

[5] A. W. Chao and M. Tigner, Handbook of Accelerator Physics and Engineering (World Scientific, Singapore, 1999).

[6] S. Adam, in Proceedings of the 14th International Cyclotron Conference (Cape Town, 1995) (World Scientific, Singapore, 1996), p. 446. 